\title{
Modelling Collaboration using Complex Networks
}

Christopher Durugbo*, Windo Hutabarat, Ashutosh Tiwari and Jeffrey R. Alcock

School of Applied Sciences,

Cranfield University,

Bedfordshire,

MK43 0AL

United Kingdom

Corresponding author email: c.durugbo@ cranfield.ac.uk

Telephone: +44 (0) 1234750111 Ext 5656 


\title{
Modelling Collaboration using Complex Networks
}

\begin{abstract}
Collaboration means working together to achieve a common goal or to solve a problem, and in modern businesses, it is an important factor for information sharing and quality. This is due to the ability of collaborations to shape the structure and behaviour of organisations through the pooling of expertise and standardising of work patterns.

Grounded on complex network theory and collaborative design research, a mathematical model of information flow for analysing collaboration in organisations is proposed in this article. The model defines concepts for characterising organisational structures for collaboration and proposes indicators for assessing organisational behaviour in terms of collaboration within organisations. The article concludes by discussing the applications and limitations of the proposed model.
\end{abstract}

Keywords: Network analysis; Conceptual modelling; Social networks; Information flows; Organisational design 


\section{INTRODUCTION}

\subsection{Research background}

Complex networks in scientific research have proven to be useful paradigms/disciplines for delineating organisations. This is due to on-going studies and renewed interests in organisational/network theory that are driven by the proliferation of web-based systems and technologies such as: e-mail, peer-to-peer and grid computing, video-conferencing and mobile/broadband connectivity $[2,11,45,52]$. Consequently, complex network concepts have been used to analyse organisational characteristics such as hierarchies $[3,26]$ and decision making [14].

Collaboration, although not a new organisational characteristic, has become a critical factor that determines the success of businesses (profit-driven organisations) [4]. It means working together in group(s) to achieve a common task or goal [4, 27, 44] and irrespective of geographical separation [2, 20, 49]. This task or goal is often beyond the capabilities of the participants involved in the collaboration. Within collaborations, participants closely work together based on durable relationships and strong commitments to a common goal with a view to pooling expertise and standardising operations [24, 45].

\subsection{Aim of paper}

In this article, collaboration is analysed in organisations as complex networks. The aim of this article is to propose a mathematical model that analyses how individuals in organisations work together to solve a problem or achieve a common goal. In order to accomplish this, the article will identify existing research that study organisations as complex networks, analyse characteristics of collaboration and propose modelling concepts for assessing the level of collaboration in organisations.

\subsection{Organisations as complex networks}

A complex network can be described as a graph $G=(V, E)$ containing a set of vertices $V$ (called nodes or points) that are associated by edges $E$ (called links or lines) [5] as shown in Fig. 1. The vertices represent entities within a network whereas edges indicate interactions based on relationships in which the entire graph is connected (i.e. for a vertex $i$ in the graph, there is a path made up of edges to another vertex $j$ ) or disconnected. A complex network can contain a subgraph $\left(G^{\prime}\right)=\left(V^{\prime}, E^{\prime}\right)$ - a subset of $G$ where $V^{\prime}$ and $E^{\prime}$ are subsets of $V$ and $E$ respectively. In Fig. 1, subgraphs can be created between sets of vertices (A, B, C, D), (A, D, $\mathrm{G}),(\mathrm{B}, \mathrm{C}, \mathrm{D}, \mathrm{F})$ and so on.

Vertices, edges and topology (that depicts how vertices and edges are arranged) are the main concepts used to characterise information structures for analysing domains such as the World Wide Web, social networks, brain networks and genetic networks [5, 33].

The mindset of 'an organisation as a network' is widely considered in research as a useful approach for promoting organisational flexibility and adaptability, particularly in the quality and sharing of information [34]. It is for this reason that complex networks can offer useful insights into how people work together based on media choice (depending on the context and needs of information flow), and communication media that influence information sharing [16, $17,37,31]$. 
Within complex network research, social network analysis (SNA) is the main approach adopted by researchers to study and understand relationships, social roles and social structure in organisations $[2,5,18,19,38,47,50]$. Examples of the use of SNA in characterising organisations include friendship networks for informal interactions and friendships [12, 32] and hierarchical networks for filling administrative layers [3, 26].

Whilst the concept of organisations as networks is viewed by some social network researchers as a 'counter-model' to the bureaucratic organisation $[2,34,36]$, other authors have argued and shown how organisations, at least for administrative purposes, can be analysed as hierarchical networks [3, 26].

SNA is often associated with organisation theory [29] and is used to identify clear patterns of relations and involvement (centralized and decentralized) based on gathered data such as the age, gender, and race of actors $[2,18,29]$. It makes use of techniques from sociology and mathematics for the representation and quantification of an organisation's information structure $[19,29]$. Although networks can be represented as a matrix or a graph, most researchers prefer graph representations in which vertices represent actors within networks, and edges indicate the relationships between the actors with a view to improving processes and performances [33, 42]. However, the use of the term 'actor' is open to the interpretation of researchers. For instance, Pryke and Pearson [36] used the term actor to represent a 'roleholding firm' whereas Van Der Aalst et al. [43] applied the term actor as individuals within an organisation.

Quantitatively, SNA is based on sociocentric (whole) approaches in which groups and group interactions are studied, and egocentric (personal) approaches in which an individual and an individual's interaction is assessed [9, 18, 42]. Sociocentric and egocentric approaches are primarily studied through cohesion and centrality respectively for characterising the information behaviour of social networks $[19,38]$.

Cohesion is a network attribute that characterises the structural interconnectedness of two vertices $i$ and $j$ in a network and is assessed in terms of: distance between vertices computed as the sum of edges along the shortest path between $i$ and $j$, reachability between vertices that establishes if $i$ and $j$ are linked directly or indirectly, and density between $i$ and $j$ that compares number of actual edges to the number of possible edges. Centrality is a network attribute that characterises the structural prominence or importance of a vertex $i$ within a network and is evaluated with regards to: degree centrality that is computed as the number of directly connected vertices to $i$, closeness centrality that is measured as the inverse of the distance between $i$ and network vertices, and betweenness centrality that is calculated as the amount of times $i$ connects other vertices to each other. These quantitative concepts offer a useful avenue for giving exact meanings and mathematical definitions for terms that ordinarily can only be described metaphorically using phrases such as 'social role' and 'prominence' [29].

\subsection{Research motivation and focus}

Renewed research interest in collaboration for organisations is motivated by the awareness that modern day business are increasingly becoming dependent on cumulative knowledge of key stakeholders such as customers and staff, for maintaining firm competitiveness and information sharing $[2,11,13,18,22,39,47,48]$. Collaboration studies in recent years has 
also been motivated by increasing informal interactions that causes companies to adopt flatter and flexible structures $[4,11]$ and the need to explore and integrate differences of team members and groups within an organisation $[11,40]$. These differences stem from preexisting work patterns and expectations of quality and success from collaborating groups and individuals. For this reason, collaboration is conceptually designed based on the use of agentor web-based systems $[39,45]$. Web based systems make use of a client/server architecture whereas agent based systems involve networks of problem solvers working together to solve problems that are beyond their individual capabilities.

Within the context of social network analysis, collaboration theory has so far centred on intra- and inter-organisational collaboration i.e. within and between organisations $[11,18,22$, 25, 28]. For instance, Cross et al. [11], in a study of 40 informal networks within 23 organisations, studied how collaboration can exist across functional and hierarchical dimensions, and concluded that informal networks are an important avenue for enabling collaboration within an organisation. Focusing on inter-organisational relationships, White [46] formulated the idea of collaborative advantage for synergies that emanate from collaborative activities and collaborative inertia related to the outcome that determines collaboration progress or advantage. Other forms of research for inter-organisational collaboration include: scientific collaboration networks [32] formed as a result of connecting papers that are co-authored by two or more scientists at different institutions and transorganisation development for improving collaboration between partner organisations [9], and inter-organisational relationships that assesses the level and performance of partnerships between organisations $[22,25]$.

With the exception of few studies such as Chinowsky et al. [8], Cross et al. [11] and White [46] that base collaboration on a subjective assessment of actors, literature suggests an absence of quantitative indicators for assessing collaboration. Rather studies within SNA have examined or proposed models that directly or indirectly influence the level of collaboration within an organisation $[2$, 17-19, 22, 25, 29, 34, 36, 42, 43, 46]. However, providing quantitative indicators for complex networks offers potentials for guiding researchers and industrial practitioners in monitoring the evolution of the organisational characteristics at intra-organisational (individual or group) and inter-organisational levels [17]. Table 1 summaries some of the related literature on modelling collaboration using social network analysis.

The focus of this paper is to make use of complex network concepts to: (i) define topologies, vertices and edges for the information structure of intra-organisational collaboration and (ii) propose quantitative indicators for the information behaviour that can be used to characterise collaboration in organisations. Key collaboration characteristics will be derived from collaborative research literature and used to propose concepts for analysing collaboration in organisations. Using a case scenario from literature, the applications and limitations of the proposed concepts will then be highlighted and discussed.

The paper plans to contribute to knowledge by: (i) proposing a model of information flow for analysing collaborations in organisations and (ii) demonstrating the use of the model in a case scenario. 


\subsection{Structure of paper}

The remainder of the paper is organised as follows: $\S 2$ describes the research method, $\S 3$ introduces the concepts for a mathematical model that can be used to analyse collaboration in organisations and a case scenario that demonstrates the use of the model whereas $\S 4$ discusses some applications and limitations of the proposed model.

\section{RESEARCH METHOD}

An analytical, applied research methodology [23] was adopted for this research. The research began analytically to capture and evaluate the characteristics of collaboration, and the information used during this evaluation was derived from a review of literature. Using the collaboration characteristics as a set of criteria, the current state of social network analysis research was evaluated and the identified gaps were then identified as 'modelling goals' for proposing the mathematical model.

The analytical, applied approach adopted in this paper is typical of model development approaches such as López et al. [26] that proposed the coordination degree model for hierarchical networks and Ehsani et al. [14] that proposed decision networks. In these approaches, researchers have extended or formulated new aspects of existing network models.

Driven by the adopted research methodology, the mathematical model development was carried out in four main stages: characteristics identification, social network analysis evaluation, model conceptualisation and demonstration, as shown in Fig. 2.

\subsection{Characterising collaboration}

The first step in the development of the model focused on identifying collaboration characteristics as seen through the perspective of collaborative design research. To determine these characteristics, key articles of collaborative design (based on citation within SCOPUS an online database for literature accessible via www.scopus.com), relevant to this work, were sourced using keywords 'collaborative design'. Of the top ten cited articles returned by the search, seven were relevant to this work. The review paper on collaborative design by Wang et al. [45] was also analysed to capture key characteristics of collaboration. The idea behind this search and analysis of articles was to ground the model within collaborative design research. Based on the search, the following collaboration characteristics were summarised from literature:

C1.Collaboration requires a network in which individuals/ groups are interconnected [35, $45,50]$ i.e. a social network

C2.Collaboration requires a network in which tasks/processes are linked [21, 45] i.e. an activity network

C3. Collaboration is required to explore and integrate differences of group members who take part in solving problems of allocated tasks that contribute to a common goal [39, $40,50]$

C4.Collaboration is closely connected and dependent on decision making, teamwork, and coordination that typify relationships and communication roles [24, 40, 41, 50] 
Coordination involves harmonising interactions between individuals to achieve a common goal [9] while decision making refers to how choices are made based on rules and procedures $[9,36]$. Teamwork involves pooling skills and resources [44] and forms the basis for collaboration within organisations [4].

\subsection{Social network analysis evaluation}

In Table 2, a set of modelling goals based on the collaboration characteristics identified in $\$ 2.1$ was used to assess the current state of social network analysis. Using SCOPUS, a search for articles with keywords 'collaboration' and 'social network analysis' returned 18 related articles that were analysed to determine the focus and current implementations in research that relate to the set of criteria. The evaluation demonstrated that no visualisation for linked processes and indicators for coordination, decision making and teamwork, within the context of this research, were available in social network analysis research. In addition, the analysis showed that current models were inadequate for characterising formal relationships that symbolise collaboration roles and responsibilities.

These formal relationships are defined by formal work practises for which tasks and events need to be defined particularly for process-intensive organisations $[6,16,21]$ and information is usually stored in a more structured form [43]. It is for this reason, that existing structures studied in SNA may not be enough to model collaboration. Nevertheless, the SNA is a flexible approach in which basic SNA concepts can be adapted by researchers to propose new attributes/indicators to characterise phenomena and systems [36]. Consequently, for the model proposed in this paper, the SNA approach has been augmented with adapted techniques from other domains and novel indicators for characterising collaboration.

\subsection{Model conceptualisation}

The next phase in the research involved making use of the identified collaboration characteristics to conceptualise the mathematical model. To do this, two main derivatives (D1 and D2) were identified by the authors of this paper based on the analysis of literature in $\S 2.1$ that produced C1-C4:

\section{D1.Intra-organisational collaboration information structure consists of: social and} activity networks (C1, C2 and C3),

From this derivative, the main information structure concepts for analysing collaboration were then obtained as a combination of social vertices and edges for individuals/groups ( $\mathrm{C} 1$ and $\mathrm{C} 3$ ), and activity vertices and edges for tasks/processes (C2 and C3).

To derive topologies of the social network for collaboration, some possible configurations for the dictator, mutual and exclusive collaboration forms captured in Maher et al. [27], and were investigated and adopted to: (i) illustrate the potential use of the model, (ii) simplify the model, and (iii) align the model with existing collaborative design research.

The topologies of the activity network in the proposed model for collaboration were based on the activity-on-node (AON), a traditional activity network employed in the widely used Project Evaluation and Review Technique and critical path method [51]. Its selection for use in the model was based on the popular use of the AON in the design and management of collaboration related tasks such as organisational projects. 
Activity-on-node representation makes use of dependencies for organising activities according to two main configurations: series and parallel configurations [10]. Dependencies exist if subsequent activities must wait for preceding activities to finish. In addition, within activity-on-node representations, a process occurs once with no feedbacks or loops [10]. To conceptualise formal relationships that symbolise roles and responsibilities, the authors of this paper have introduced a set of edges for interfacing social vertices with activity vertices. The introduced 'interface edges' represent relationships that are associated with individuals, teams and organisations for involvement in linked processes that contribute to a common goal.

\section{D2.Intra-organisational collaboration requires indicators for authority (decision} making), teamwork, and coordination within topologies, vertices and edges (C3 and C4).

Based on this derivative, a set of novel indicators for collaboration was proposed by the authors, and to compute each indicator a constant is introduced to quantify the strength of network relationships and the availability of collaboration information. The introduced constants are as follows: coordination constant $\left(\alpha_{i}\right)$, decision constant $\left(\beta_{i}\right)$ and teamwork constant $\left(\gamma_{i}\right)$. These constants are subjective probabilities that are based on the availability of a vertex $i$ to: harmonise interactions $\left(\alpha_{i}\right)$, make choices $\left(\beta_{i}\right)$, and pool resources $\left(\gamma_{i}\right)$. The proposed collaboration indicators for a vertex $i$ include: decision-making scale $\left(\delta_{i}\right)$, coordination scale $\left(\chi_{i}\right)$ and teamwork scale $\left(\tau_{i}\right)$. These identified indicators are consistent with existing studies in complex network research where decision making measures have been introduced for agent-based systems [14] and coordination quantities for edges between vertices have been investigated for hierarchical networks $[3,26]$.

The proposed collaboration indicators are introduced because existing quantities identified in literature have been used in different contexts to those defined in \$2.1 for decision making, teamwork and coordination. For instance, the coordination degree by López et al. [26] measures the ability of a vertex $i$ to interchange information with another vertex $j$ within a network and the coordination score by White [46] assesses the degree to which networks are concentrated around important vertices.

The indicators as shown in Fig. 3 are derived as sums of existing SNA measures for clustering coefficient, closeness and degree centrality. These quantities were selected because they reflect interconnectedness within groups, individual connections for relationships and activity of individuals respectively $[18,42]$.

The degree centrality $\left(D c_{i}\right)$ is a ratio of number of directly connected vertices to the number of possible vertices in a network and can be computed as:

$$
D c_{i}=\frac{\operatorname{leg}_{i}^{-}}{N-1}
$$

Where, $\mathrm{N}$ is the number of vertices in the network and $[\mathrm{deg}]_{i}$ is the number of vertices directly connected to $i$.

The clustering coefficient $\left(C c_{i}\right)$ assesses the density between vertices and represents the tendency for vertices to cluster together. If a vertex $i$, connects to $b_{i}$ neighbours, and the 
number of possible edges between the vertices is given as $b_{i}\left(b_{i}-1\right) / 2$, then $C c_{i}$ for $i$ can be computed as:

$$
C c_{i}=\frac{2 n_{i}}{b_{i} \boldsymbol{Q}_{i}-1}
$$

Where $n_{i}$ is the number of edges between $b_{i}$ neighbours.

The closeness $\left(c_{i j}\right)$ between vertices defines the order with which one vertex connects to another vertex. It is computed as the inverse of the geodesic distance $\left(d_{i j}\right)$ between a pair of vertices $i$ and $j . d_{i j}$ is the number of edges along the shortest path between $i$ and $j . c_{i j}$ can be calculated as:

$$
c_{i j}=\frac{1}{\sum_{i \neq j \in N} d_{i j}}
$$

For instance, if an individual connects directly to another collaborator (i.e. participant in a collaboration), the closeness is given as 1 , if an edge is established as a result of connecting to a third vertex $k$ acting as a hub or by dictator collaboration [45], then vertex $i$ has a closeness of 0.5 to vertex $j$.

In the proposed model, configurations proposed in D1 were used to develop eqns. (4-7) that analyse the information structure for social and activity networks. Eqns. (4-7) were then used in combination with eqns. (1-3) to formulate the collaboration indicators of eqns. (8-13).

\subsection{Scenario demonstration}

To demonstrate the use of the model for analysing collaboration in organisations, an example from literature [15] was presented and analysed. The example was selected for familiarisation and simplicity purposes. In the demonstration, case scenarios of collaboration will be generated from the example and possible topologies, vertices and edges based on the proposed model will be investigated. Coordination, team-work and decision making indicators for each case scenario will then be compared.

The proposed model will also be assessed against the collaboration characteristics criteria identified in $\S 2.1$.

\section{AN INTRA-ORGANISATIONAL COLLABORATION MODEL}

Intra-organisational collaboration (IOC), in this paper, is modelled as a connected, partitioned, non-overlapping hypergraph $G=(V, E)$ containing a graph for characterising the collaborative social network of individuals/groups $G_{s}=\left(V_{s}, E_{s}\right)$ and a digraph for characterising the collaborative activity network of processes/tasks $G_{p}=\left(V_{p}, E_{p}\right)$, as shown in Fig. 4. $V_{s}$ represents social vertices of collaborating individuals, teams or organisations, and $V_{p}$ represents activity vertices for processes that are required to achieve a common goal that could not be achieved by the collaborating individuals. $E_{s}$ and $E_{p}$ correspond to edges between teams (or individuals) and processes. 
For the proposed model, processes become part of a collaboration based on the set of interface edges $T$ created by vertices within collaborators i.e. $T$ associates $V_{s}$ with $V_{p}$. Interface edges are connections between individuals/groups and tasks/processes for the exchange of resources. For instance, a machine operator may work on a problem and exchange information with a piece of equipment as part of a process in an intra-organisational collaboration. This interaction, related to formal work practise, can be enabled by edges (defined here as interface edges) for human-machine relationships. Each social vertex can be linked to as many as $V_{p}$ activity vertices.

The maximum number of possible interface edges in the model is given as $V_{s} \times V_{p}$ in which every social vertex is linked to every activity vertex. Consequently $G$ is defined by $V=V_{s} \mathrm{U}$ $V_{p}$ and $V_{s} \cap V_{p}=\varnothing$. Similarly, $E=E_{s} \cup E_{p} \cup T$ and $E_{s} \cap E_{p} \cap T=\varnothing$.

This section makes use of complex network concepts and properties to characterise the underlying topology, composed vertices and connected edges of the IOC model.

\subsection{Collaboration social network}

For $f$ groups (each containing $g$ social vertices) within the social network $G_{s}$, three different (Types 1 to 3) topologies for characterising IOC are proposed as shown in Fig. 5.

In Type 1 topologies, based on dictatorship, collaboration between groups and individuals is realised by means of a leading hub in each group that is appointed to dictate or dominate interactions for collaborations between individuals and groups. In the proposed configuration, collaborating spokes within an organisation are connected to the group hubs (i.e. as a star or hub-and-spokes arrangement).

For a group containing a single hub, the social network contains $g-1$ spokes that are connected to the hub. The total number of hubs that enable collaboration in multiple groups is given as $f$ while the total number of spokes within $f$ groups is given as $f(g-1)$ i.e. $f g-f$. Type 2 topologies, motivated by mutual collaboration, enable edges between connected social vertices who occupy themselves working with other social vertex in a group to achieve a specific goal that is posed. Also, groups are connected by a 'connector hub' that maintains collaboration across groups. Within a type 2 topology containing $f$ groups, $f g-f$ social vertices (or spokes) can link with $f$ hubs with connector roles. Each vertex within a group can also connect to other vertices within its group (i.e. $g-1$ vertices) to work on a separate part of a problem that contributes to a common goal.

Type 3 topologies involve exclusive collaboration and enable edges between connected social vertices (that act as hubs) with similar or dissimilar specialties. Each social vertex works on achieving a collaborative goal and occasionally connects and negotiates with other vertices across collaborating groups for advice and updates on the status of factors such task prerequisites and dependencies, and to solve by uni-, inter- or multi-disciplinary problems. In the type 3 topology, collaboration is based on exclusive roles and the number of collaborating teams across organisations is equal to $f$ whereas collaboration is enabled by maximum of $V_{s}$ collaborating vertices.

In all the forms of social network topologies proposed in the IOC model, the number of vertices within the social network $\left(G_{s}\right)$ can be calculated as the sum of social vertices from each group i.e.: 


$$
\left|V_{s}\right|=\sum_{i=1}^{f} g_{i}
$$

Where $\left|V_{s}\right|$ is the cardinality of $V_{s}, f$ is the number of groups involved in collaboration and $g_{i}$ is the number of social vertices that form a group $i$.

Within $G_{s}$, as shown in Fig. 5, two forms of edges facilitate connections: collaborative- and network- edges.

Collaborative-edges $\left(E_{s}^{\prime}\right)$, shown in Fig. 5 as gray coloured lines between vertices, are a subset of edges that form a sub-graph of the social network $\left(G_{s}{ }^{\prime}\right)$ for enabling collaboration between groups. Within the type 1 and 2 topologies, $f$ social vertices across teams (interteam) acting as hubs can form $f(f-1) / 2$ collaborative edges with each other. In the type 3 topology, each social vertex exclusively collaborates (i.e. creates edges) across groups by establishing $g \times f(f-1) / 2$ edges based on factors such as common disciplines or pre-defined problems,.

Network edges $\left(E_{s}\right)$ on the other hand, are possible edges for the topologies shown in Fig. 5, and their cardinality $\left|E_{s}\right|$ are computed as follows:

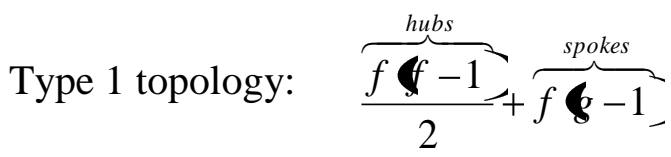

Type 2 topology: $\overbrace{\frac{f \boldsymbol{f}-1}{2}}^{\text {hubs }}+\overbrace{\frac{f g(\boldsymbol{g}-1}{2}}^{\text {chains }}$

Type 3 topology: $\quad \overbrace{\frac{f g(t h i n-1}{2}}^{\text {witroups }}+\frac{\overbrace{g f(\mathbb{f}-1}^{\text {across-groups }}}{2}=\frac{1}{2} \coprod^{2} f-2 f g+f^{2} g$.

\subsection{Collaboration activity network}

The activity network $G_{p}$ within the IOC model is derived from: serial topologies that impose precedence in dependencies for creating an additive chain of processes, and parallel topologies that enforce multiple dependencies for concurrent processes. The parallel topology may involve multiple processes that are dependent on a single process (burst) or a single process that is dependent on multiple processes (merge) as shown in Fig. 6.

For an activity network $\left(G_{p}\right)$ contains $I$ and $J$ number of serial and parallel configurations for vertices, the number of vertices within $G_{p}$ i.e. $\left|V_{p}\right|$ can be computed as:

$$
\left|V_{p}\right|=\overbrace{\sum_{i \in I}^{I} s_{i}}^{\text {serial }}+\overbrace{\sum_{j \in J}^{J} p_{j}}^{\text {parallel }}
$$

Where, $s_{i}$ and $p_{j}$ are the number of processes in each serial and parallel configuration respectively and $\left|V_{p}\right|$ is the cardinality of $V_{p}$. Suppose an intra-organisational collaboration is set up to carry out $4,3,5$ and 2 processes with parallel dependencies and 9 serially dependent 
processes, and if the IOC makes use of 5 collaborating teams each containing 6 team members, then the number of vertices within the IOC will be 53 , broken down as $5 \times 6=30$ social vertices for $G_{s}$ and $(4+3+5+2)+9=23$ activity vertices for $G_{p}$ If the activity network is made up of $I$ serial, $L$ parallel (burst) and $M$ parallel (merge) then processes within $G_{p}$ of the IOC are associated by $E_{p}$ input and output edges in the formulation:

$$
\left|E_{p}\right|=\overbrace{\sum_{i=1}^{I} a_{i}^{\prime}}^{\text {serial }}+\overbrace{\sum_{l=1}^{L} b_{l}^{\prime}+\sum_{m=1}^{M} c_{m}}^{\text {parallel }}
$$

$\left|E_{p}\right|$ is the cardinality of $E_{p}, a_{i}^{\prime}$ and $b_{l}^{\prime}$ are inputs to $I$ serial and $L$ parallel (burst) sets of configured vertices and $c_{m}$ is the output edge from $M$ parallel (merge) sets of configured vertices where $E_{p}=a_{i}{ }_{i} \cup b^{\prime}{ }_{l} \cup c_{m}$ and $a_{i}{ }_{i} \cap b_{l}{ }_{l} \cap c_{m}=\varnothing$. The maximum number of edges within $G_{p}$ can be computed as $V_{p}\left(V_{p}-1\right) / 2$. However, when $L=0$ then the maximum number of edges within $G_{p}$ can be simplified to $2 V_{p}-2$ activity edges. Two edges are subtracted from the total number for terminal vertices- the start vertex that has no preceding vertices and the end vertex that has no following vertices.

\subsection{Collaboration indicators}

Within the IOC network (i.e. $G_{s}$ and $G_{p}$ ), three collaboration indicators with values greater than or equal to zero and less than or equal to two are proposed.

The first indicator termed the 'teamwork scale' $\left(\tau_{i}\right)$ is introduced to assess the activity of a social vertex $i$ and interconnectedness within a cluster for teamwork. To do this, the degree centrality and clustering coefficient of $i$ are multiplied by a teamwork constant $\left(\gamma_{i}\right)$ that is based on the availability and capability of $i$ (i.e. the participant) to pool resources. The teamwork scale $\tau_{i}$ can be calculated as:

$$
\text { For a social vertex } i \quad \tau_{i}=[\overbrace{\frac{2 E_{s}}{V_{s}^{\prime} \boldsymbol{\mho}_{s}^{\prime}-1}-}^{\text {clust_coefficient }}+\frac{\overbrace{T_{\text {eg }}}^{\text {deg_centrality }}}{V_{s}^{\prime}-1}] \cdot \gamma_{i}
$$

Where, $\left[\mathrm{deg}^{\mathrm{s}}\right]_{i}$ is the number of social vertices that are directly linked to $i$. For the overall IOC network, the average teamwork scale $(\tau)$ can be calculated as:

$$
\tau=\frac{1}{V_{s}^{\prime}} \sum_{i=1}^{V_{s}^{\prime}} \tau_{i}
$$

Where, $V_{s}^{\prime}$ is a sub-graph consisting of social vertices at group, inter-group or organisational level. 
The 'decision-making scale' $\left(\delta_{i}\right)$ is the second collaboration indicator introduced to assess the ease with which a social vertex $i$ within the intra-organisational network can make decisions based on the interconnectedness and connections for relationships. To do this, the clustering coefficient and closeness of $i$ in a defined sub-graph (group or overall organisation) of the collaboration social network are multiplied by a decision constant $\left(\beta_{i}\right)$ that is dependent on the availability and capability of $i$ to make choices. It is calculated as:

$$
\text { For a social vertex } i \quad \delta_{i}=[\overbrace{\sum_{i \neq j \in V_{s}^{\prime}} d_{i j}}^{\text {closeness }}+\frac{\overbrace{2 E_{i}}^{\text {clust_coefficient }}}{V_{s}^{\prime} \boldsymbol{\mho}_{s}^{\prime}-1}] \cdot \beta_{i}
$$

Where, $d_{i j}$ is the distance between two vertices $i$ and $j, E_{i}$ is the number of edges created with directly connected vertices. The average decision-making scale $(\delta)$ for social vertices in the IOC network can then be computed as:

$$
\delta=\frac{1}{V_{s}^{\prime}} \sum_{i \in V_{s}^{\prime}}^{V_{s}^{\prime}} \delta_{i}
$$

The third indicator, the 'coordination scale' $\left(\chi_{i}\right)$ assesses the connections and activity associated with which a social vertex $i$ through which interactions can be harmonised. To do this, a coordination constant $\left(\alpha_{i}\right)$ that is dependent on the availability and capability of $i$ for harmonising interactions, is multiplied by the sum of the closeness and degree centrality of $i$ towards the social and activity network. The activity network is included to take into account coordination theory that depicts dependencies as emerging from tasks [1]. The coordination scale $\chi_{i}$ can be calculated as:

For a social vertex $i$

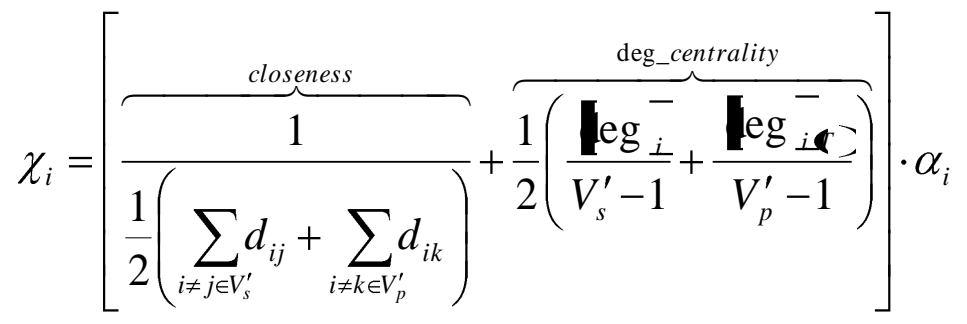

Where, $V_{p}{ }^{\prime}$ is a sub-graph consisting of activity vertices and $\left[\mathrm{deg}^{\mathrm{s}}\right]_{i(T)}$ is the number of activity vertices that are directly linked to $i$ through interface edges that constitute $T$. The average coordination scale $(\chi)$ for social vertices in the IOC network can then be computed as:

$$
\chi=\frac{1}{V_{s}^{\prime}} \sum_{i \in V_{s}^{\prime}}^{V_{s}^{\prime}} \chi_{i}
$$

\subsection{An example: intra-organisational collaboration for product development}

Eppinger [15], adapted to exemplify the application of the proposed model in this paper, is based on the management of the development of power trains at General Motors. No 
indication is given as to the social network for collaborating teams or the number of members in each team, rather the focus of Eppinger [15] was to make use of the design structure matrix approach to analyse the sequence and configuration of processes based on the frequency of information flow feed-forwards and feed-backs.

The frequency of communications involving information flow, centred on daily, weekly and monthly interactions and the main design challenge was to improve communications for systems integration. This challenge was dealt with by reorganising the information flow through the introduction of a systems integration team and four new 'overlapping' teams. Teams were overlapped based on the sequence of processes and regular team interactions.

\subsubsection{Pre-existing information flow}

Prior to reorganisation, as shown in Fig. 7a and Table 3, the intra-organisational collaboration for product development (IOC-PD) was made up of four teams that deliver short block systems (SBS), valve train systems (VTS), induction systems (IS), and emissions and electrical systems (EES). The IOC-PD is also made up of 22 processes i.e. an activity network made up of 22 activity vertices (A to V), assigned as follows: the SBS team was responsible for developing engine blocks (A), crankshafts (B), flywheels (C), pistons (D), connecting rods (E) and lubrication (F), the VTS team was responsible for cylinder heads (G), camshaft/valve trains (H), and water pump/cooling (I), the IS team was responsible for intake manifold (J), fuel system (K), accessory drive (L), air cleaner $(\mathrm{M})$, AIR $(\mathrm{N})$ and throttle body $(\mathrm{O})$, the EES team was responsible for exhaust (P), EGR (Q), EVAP (R), ignition (S), ECM (T), and electrical system (U), while all collaborating teams were responsible for engine assembly $(\mathrm{V})$. This demonstration assumes that each team the initial IOC-PD is made up of five members corresponding to 20 human collaborators i.e. a social network made up of 20 social vertices. Five is chosen for this demonstration because it is the minimal value of the magic number for group sizes that is widely accepted as seven plus or minus seven [6].

Table 4 presents the main results of the IOC-PD demonstration. The table provides data on the number of nodes, groups, participants, hubs and spokes (where appropriate DERIVED FROM the description of the scenario. Using these values and topologies from Fig. 7, the values for SNA measures (clustering coefficient, degree centrality and closeness) were then computed. The last nine rows of Table 4 present the collaboration indications (individual and average) based on the calculated SNA measures.

The first step in determining the collaboration indicators involves calculating the SNA measures of the network.

\section{Clustering coefficient}

Whereas the maximum number of vertices in a fully connected social network for the IOCPD can be computed as $f g(f g-1) / 2$ i.e. 190, the hubs and spokes in Type 1 topologies can form $((f(f-1) / 2)+(g-1))$ and $(g-1)$ actual edges respectively as shown in Fig. 7a. The clustering coefficient $\left(C c_{i}\right)$ for each hub and spoke in the Type 1 topology can then be computed as $((4(4-1) / 2)+(5-1)) / 190=0.0526$ and $(5-1) / 190=0.0211$ respectively. For Type 2 and 3 topologies, each hub and spoke can have $((f(f-1) / 2)+(g(g-1) / 2))$ and $g(g-1) / 2$ actual edges corresponding to $C c_{i}$ values of 0.0842 and 0.0526 respectively. 
Degree centrality

Within the social network of the IOC-PD, each hub would have $(f-1)+(g-1)$ i.e. 7 neighbours whereas the spokes would have 1 neighbour (the dictator hub) in the Type 1 topology and $g-1$ i.e. 4 neighbours in the Type 2 topology. From eqn. (1) and Fig. 7a, the degree centrality $\left(D c_{i}\right)$ for hubs can then be computed as $7 /(20-1)=0.3684 . D c_{i}$ for spokes can be calculated as $1 /(20-1)=0.0526$ and $4 /(20-1)=0.2105$ for Type 1 and Type $2 / 3$ topologies respectively.

Within the Type 1 topology, $D c_{i}$ for social vertices within the entire network of social and activity vertices can be calculated, using the interface edges shown in Table 3, as follows:

\begin{tabular}{lll} 
For SBS, IS and EES teams & $\begin{array}{l}\text { (Hubs) } \\
\text { (Spokes) }\end{array}$ & $\begin{array}{l}(7+7) /((20-1)+22)=0.3415 \\
(1+7) /((20-1)+22)=0.1951\end{array}$ \\
\hline \multirow{2}{*}{ For VTS team } & $\begin{array}{l}\text { (Hubs) } \\
\text { (Spokes) }\end{array}$ & $\begin{array}{l}(7+4) /((20-1)+22)=0.2683 \\
(1+4) /((20-1)+22)=0.1220\end{array}$
\end{tabular}

Values for $D c_{i}$ in Type 2 and 3 topologies have been computed using similar approaches and are shown in Table 4.

\section{Closeness}

Within the social network of the IOC-PD, the value of $d_{i j}$ : between two hubs is 1 , between a hub and a spoke in the hub's team is 1 , between a hub and a spoke in a different groups is 2 , between two spokes in a different group is 3, and between two spokes in the same group is 2 for Type 1 and 1 for Type 2 topologies. The geodesic distance for social vertices within the social network can therefore be computed as follows:

\begin{tabular}{|c|c|}
\hline For each hub: & $\overbrace{(\times(f-1)}^{\text {hub-hub(across_group.s. }}+\overbrace{(\times(g-1)}^{\text {hub-spoke(same_group) })}+\overbrace{(\times \times(f-1) \mathbf{8}-1}^{\text {hub-spokeddifferent_group })}$ \\
\hline $\begin{array}{l}\text { For each spoke in } \\
\text { Type } 1 \text { topology: }\end{array}$ & 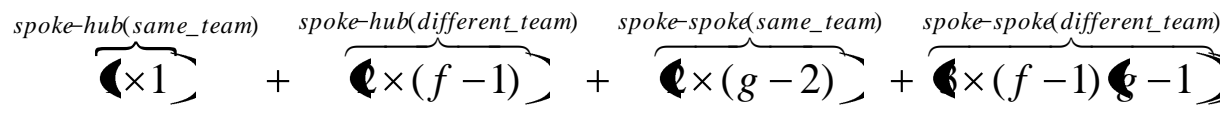 \\
\hline $\begin{array}{l}\text { For each spoke in } \\
\text { Type } 2 \text { topology: }\end{array}$ & $\overbrace{(\times 1}^{\text {spoke-hub(same_team })}+\overbrace{(\times(f-1)}^{\text {spoke-hub(different_team })}+\overbrace{(\times(g-2)}^{\text {spoke-spoke(same_team })}+\overbrace{(\mathbf{8 \times ( f - 1 )}}^{\text {spoke-spoke(different_team })}$ \\
\hline
\end{tabular}

Similarly, $d_{i k}$ for social vertices to an activity vertex $k$ via interface edges $T$ can be calculated from the edges of topology of the activity network, shown in Fig. 7a, as follows:

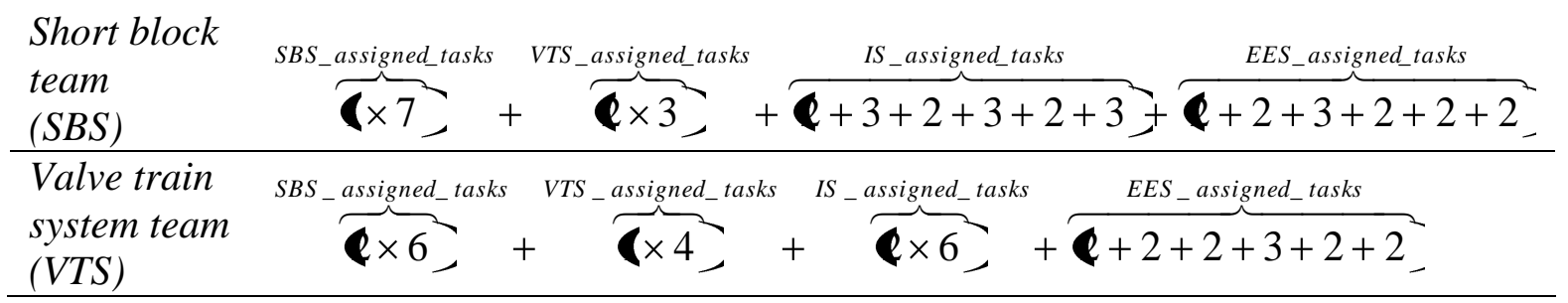




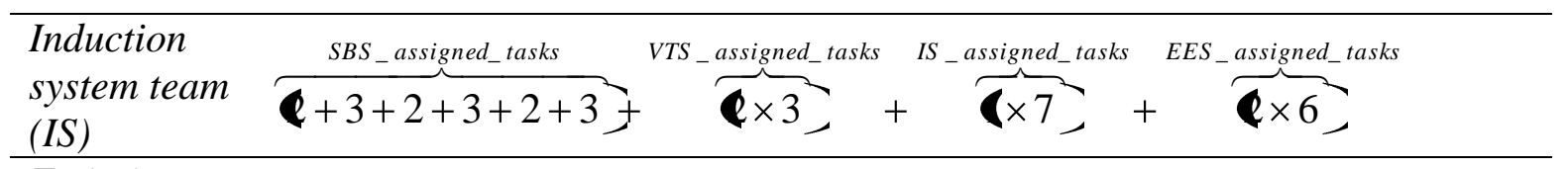

Emissions

and SBS_assigned_tasks VTS_assigned_tasks IS_assigned_tasks EES_assigned_tasks

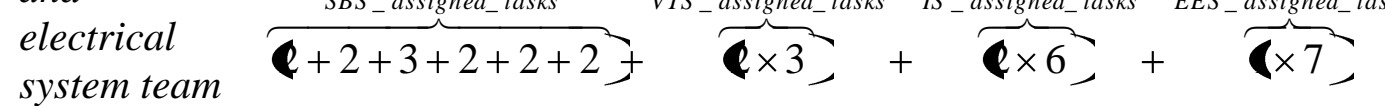

(EES)

Values for $D c_{i}$ in Type 2 and 3 topologies have been computed using similar approaches and are shown in Table 4.

Using the $d_{i j}$ and $d_{i k}$, of social vertex $i$ the closeness of $i$ within the social network and the entire IOC-PD network can be computed as shown in Table 4.

\section{Collaboration Indicators}

The next step in deriving the collaboration indicators involves multiplying the different SNA quantities with the various constants proposed in the model.

Assuming each vertex is always available and capable to harmonise interactions, pool resources and make choices, i.e. $\gamma_{i}, \alpha_{i}$ and $\beta_{i}$, are all 1 , then the various collaboration indicators can be calculated, using Fig. 7a and eqns. (8-13), as shown in Table 4.

The table shows that the most effective means for collaboration was the Type 3 topology with $0.4526(22.6 \%), 0.1165(5.8 \%)$ and $0.3513(17.6 \%)$ out of a possible value of 2 for teamwork, decision-making and coordination.

\subsubsection{Reorganised information flow}

Following the reorganisation, the old IOC-PD configuration is replaced with four new teams (numbered 1 to 4 ) and an integration team. In the new IOC-PD, the teams are assigned to 6 , 7, 8 and 5 tasks respectively with multiple teams working on the few overlapping processes as shown in Table 5 and Fig. $7 \mathrm{~b}$. The integration team is exclusively assigned to five processes L-V.

In Eppinger [15], the reorganisation involved the restructuring of available personnel which in this example corresponds to five groups made up of four personnel giving a total of 20 collaborating social vertices as in \$3.4.1. Using the intra-collaboration model, the updated values for collaboration indicators can be derived as shown in Table 4.

For the Type 1 topology, an additional hub and more edges between hubs due to increased number of group causes an improvement to the clustering coefficient of the social network, whereas degree centrality and closeness values remain constant. The overall effect of the reorganisation is that collaboration improves for the Type 1 topology.

For the Type 2 topology, the additional connector hub causes a decrease in the $C c_{i}, D c_{i}$ and $c_{i j}$ values for connected social vertices. This results in an overall decrease in the collaboration indicators although these values remain higher than those of the Type 1 topology.

Within the Type 3 topology, each social vertex acts as a hub meaning $C c_{i}$ and $D c_{i}$ values remain the same. However, the closeness decreases and counteracts gains due to increased coupling of processes. Consequently, teamwork and decision-making scales remain constant whereas coordination decreases slightly. Nonetheless, the Type 3 topology based on 
exclusive collaboration offers the highest values for collaboration indicators in both the preexisting and re-organised information flow, correlating with previous empirical studies such as Maher et al. [27] which suggest that exclusive collaborations are the most effective and productive.

\section{DISCUSSION}

In Table 6, the intra-organisational collaboration model proposed in this paper is evaluated based on the characteristics of collaboration identified in $\$ 2.1$, with regards to the information structure and behaviour for organisations. The table demonstrates coverage of the required characteristics for collaboration in organisations.

This section highlights and discusses some applications and limitations of the proposed model.

\subsection{Applications of the model}

The intra-organisational collaboration model proposed in this paper can be applied by researchers and practitioners in two main ways: (i) for enhancing the quality and sharing of information within organisations and (ii) for analysing roles for communication during collaboration.

\subsubsection{Enhancing information quality and sharing}

Information quality describes the free flow of information within an organisation whereas information sharing is a factor of information flow that describes the joint use of critical and proprietary information that could be generic (inventory control policies) or specific (weekly manufacturing schedule) in nature [13].

In terms of enhancing information quality and sharing, the proposed model can be used to plan the configuration of organisations through the identification and selection of suitable collaboration topologies. Possible configurations such as those identified in $\S 3.1$ and $\S 3.2$ could be generated and collaboration indicators such as those proposed in $\$ 3.3$ may then be used to analyse potentials for collaboration. This is typical of network analysis techniques that explore the paradox of peripherality versus centrality of actors in an organisation [46]. For the case scenario of the IOC-PD presented in $\$ 3.4$, the type 3 configuration scored highest in terms of potentials for teamwork, decision-making and coordination with $\tau, \delta$, and $\chi$ values of $0.4526,0.1165$ and 0.3513 respectively, as shown in Table 4 . However, the reorganised information flow for the type 3 configuration failed to improve the collaboration indicators. Rather, the coordination degree decreased by a value of $0.0003(-0.85 \%)$. In contrast, the generated values for $\tau, \delta$, and $\chi$ in the type 2 configuration decreased by 0.0458 $(-15.21 \%), 0.014(-16.91 \%)$ and $0.0145(-5.02 \%)$ respectively.

For the type 2 configuration in $\$ 3.4$, the results of comparing the pre-existing and reorganised information flow showed that generated $\tau, \delta$, and $\chi$ values increased by 0.0173 $(+12.08 \%), 0.0022(+4.39 \%)$ and $0.0075(+3.27 \%)$ respectively.

The managerial implication of the results is that enhancements to information flow must be driven by an analysis of the initial configuration of organisations. In practice, the configuration of an organisation may involve a combination of all the topologies identified in 
$\S 3.1$ and $\S 3.2$. An initial analysis of the organisation's topology is therefore required prior to reorganisation. For instance, managers could decide to change an organisation's topology from a type 1 to a type 3 configuration. Alternatively, strategies for improving information flow could be investigated and analysed. Using the proposed model for instance, alternative structures for social and activity vertices can be applied to improve intra-organisation collaboration as shown by the plots in Fig. 8. Fig. 8a depicts a chart of the average decisionmaking scales, Fig. $8 \mathrm{~b}$ shows the average coordination scales, whereas Fig. 8c presents the average teamwork scale chart. In the plots, four different configurations are applied to generate collaboration indicators for the IOC-PD. The first two markers in each chart from left to right represent the collaboration indicators for the pre-existing and reorganised information flow respectively. The third markers (that offer highest values for coordination as shown in Fig. 8b) represent changes to the pre-existing information flow achieved through high coupled processes in which each of the original four teams are assigned to 21 overlapping tasks each. The fourth markers (that offer highest values for decision-making and teamwork as shown in Fig. 8a and 8c) indicate modification to the pre-existing information flow by means of restructuring personnel to two groups made up of ten members each. In Fig. $8, \gamma_{i}, \alpha_{i}$ and $\beta_{i}$ are all 1 (i.e. vertex is always available and capable to harmonise interactions, pool resources and make choices).

In practice, the proposed collaboration indicators can vary depending on factors such as skill levels, staff knowledge and experience, working hours, study/sick leaves and involvement in multiple projects. High values of collaboration indicators for vertices towards therefore suggest high potentials for working together whereas low collaboration indicators could imply high independent work/ research. Consequently, collaboration indicators could offer a useful avenue for planning staff availability, implementing staff covers and backup, and establishing multiple information access points.

\subsubsection{Analysing communication roles}

As shown in Table 1, existing literature on modelling collaboration using social network analysis have largely concentrated on evaluating how participants communicate during collaboration. Within the proposed IOC model, human participants can take up key roles as hubs or spokes according to nature of the collaboration - dictatorship, mutual or exclusive. Within the case scenario of the IOC-PD, type 1 topology hubs share similar pre-existing $D c_{i}$ (individual connections) and $c_{i j}$ (activity) scores of 0.3684 and 0.0323 respectively, with type 2 and 3 topology hubs. However, in terms of interconnectedness, type 1 topology hubs have lower $C c_{i}$ scores of 0.0526 in comparison to the $C c_{i}$ scores of 0.0842 for the type 2 and 3 topology hubs. Similarly, as shown in Table 4 , the $D c_{i}, c_{i j}$ and $C c_{i}$ scores for the type 1 topology spokes are lower than the type 2 topology spokes.

For organisational managers, periodical assessments of $D c_{i}, c_{i j}$ and $C c_{i}$ scores could offer a useful avenue for evaluating the performance of an organisation's agent- and web-based systems. In the analysis of agent based systems multiple agents may assume the role of a single vertex and a vertex may assume multiple roles, an occurrence known as 'interlocking' that has been the focus of studies in which individuals, usually directors affiliated to one organisation, sit on the board of other organisations [30]. Similar interlocking ideas have been applied in industrial practise for design processes with a view to promoting coupled 
designs [40]. Also, in web-based systems social vertices acting as servers may be included in the social network to serve as hubs for clients. Special considerations for server-to-server edges could then be made for enhancing collaboration through the timely synchronisation of

servers across groups with minimal disruption to the availability of information. Furthermore, as shown in Fig. 8, a combination of fewer groups and coupled tasks could be combined or traded-off for improved collaboration. Consequently, layers of groups resembling hierarchical structures could be created for effective collaborative work that requires high numbers of social vertices. Where this is the case, groups of 'collaborative actors' may then become the unit for analysis for social vertices in the IOC model.

\subsection{Limitations of the model}

Although this paper identifies a single indicator for each collaboration characteristic, it is however important to note that users of the proposed model still require some training or experience in the use SNA. This is because terms such as closeness and clustering coefficient are fundamental quantities from the SNA technique. The simplicity of the proposed model has also meant that basic quantities and collaboration forms have been adopted. For instance, the degree is a measure used in the model that can be broken down further into indegree and outdegree that characterise the direction of edges between two vertices. If the degree is based on directed edges towards a vertex $i$ then it is known as the indegree whereas if it is based on directed vertices from $i$ then the measure is known as the outdegree [46]. Furthermore, in the model the presence as opposed to the strength of edges is employed. This strength of relationships accounts for why individuals with similar characteristics usually associate with one another, a trend known as homophily [49].

Also, in this paper, the proposed collaboration indicators are analysed from the perspective of social vertices within the network in accordance with the SNA technique from which the model in this paper was proposed. Furthermore, in the case scenario of the IOC-PD, it is assumed that vertices are always available and capable of establishing edges for harmonising interactions, pooling resources and making choices. However in real-world scenarios, activities may be automated or semi-automated for activity vertices to take over some collaborative work resulting in 'indirect influences' and improvements on the level of collaboration in organisations.

\subsection{Future Research Directions}

Prior works by authors such as López et al. [26] and Ehsani et al. [14] have proposed or demonstrated the use of mathematical models for analysing collaboration characteristics or relationships. Similarly, this article has proposed and demonstrated the use of a mathematical model, i.e. the IOC model, for analysing collaborations in organisations. However, the case scenario used in the demonstration was based on an example from academic literature. Consequently, challenges exist to explore the practicality and usability of the IOC model for analysing real-life organisations and processes. There is therefore a need to examine if the IOC model can be applied for specific or a wide range of companies and to define the performance of the model for effective collaborations. Some useful research areas that could be explored include the extent to which the IOC model could be applied for analysing 
collaborations, the performance of the IOC model against other tools, and the validation of the model across different organisations.

Case studies of companies could be used to capture the topologies, vertices and edges of the IOC model and to outline lessons that could be learnt and used to improve collaborations.

\section{CONCLUSIONS}

In this article, a sociological and technical (i.e. socio-technological) perspective has been applied to mathematically model an organisation as a network that collaborates to solve a problem or achieve a goal. Useful insights from the proposed 'intra-organisational collaboration model' in this paper suggested that an organisation can be: analysed as an amalgamation of social networks of human actors and activity networks of processes, and assessed through indicators for teamwork - to tally the manner in which participants and groups pool resources to achieve a goal, purposely, or inadvertently, decision-making - to score the manner in which choices are made during collaborations through dictated decisions by a dictating entity, participatory decisions made by participating entities and democratic decisions based on collaborators who are individually responsible for decision making, and coordination - to measure the ability of collaborators to harmonise interactions for maintaining and updating the flow of resources such as materials, funds and information. A case scenario of the management of the development of power trains was used to demonstrate how the mathematical model can be used to analyse collaborations within an organisation. The scenario compared the pre-existing information flow involving 4 teams of 5 members and the re-organised information flow involving 4 teams of 4 members. The analysis of the case scenario showed that for topologies based on mutuality, collaboration indicator values decreases whereas for topologies based on dictatorship, collaboration improves. However, collaboration indicator values remain constant for topologies based on exclusivity. These findings suggested that merely discovering and concentrating on working in a group may not be adequate for collaboration, there is a need to factor the number and levels of collaboration much like hierarchies in traditional organisations as well as the overlapping of tasks that may be automated or semi-automated.

Within the proposed IOC model, communication is enabled by social, activity and interface edges. For researchers and industrial practitioners, the presence of these different edges presents a wide range of communication roles for enabling human-to-human, human-toprocess and process-to-process communications. Furthermore, within the proposed IOC model, initial or regular analysis of the information structure and behaviour for collaboration can be conducted to determine and review information flow factors such as group sizes, data storage roles, and flow control policies. Also, as discussed in the paper, the proposed model can serve as a benchmarking approach for improving the free flow and exchange of information within organisations. 


\section{ACKNOWLEDGMENTS}

The authors would like to extend their sincere thanks to the Engineering and Physical Sciences Research Council (EPSRC), for its support via the Cranfield Innovative Manufacturing Research Centre (CIMRC), towards the work carried out in the preparation of this paper.

\section{REFRENCES}

[1] V. Albino, P. Pontrandolfo, B. Scozzi, Analysis of information flows to enhance the coordination of production processes, International Journal of Production Economics 75 (2002) 7-19.

[2] J.G. Anderson, Evaluation in health informatics: social network analysis, Computers in Biology and Medicine, 32 (2002) 179-193.

[3] D. Ben-Arieh, M.A. Pollatscheck, Analysis of information flow in hierarchical organizations, International Journal of Production Research 40 (2002) 3561-3573.

[4] M.M. Beyerlein, S. Freedman, C. McGee, L. Moran, Beyond Teams: Building the Collaborative Organization, Jossey-Bass/Pfeiffer, San Francisco, 2003.

[5] S. Boccaletti, V. Latora, Y. Moreno, M. Chavez, D.-U. Hwang, Complex networks: structure and dynamics, Physics Reports 424 (2006) 175-308

[6] B.G. Cain, J.O. Coplien, N.B. Harrison, Social patterns in productive software development organizations, Annals of Software Engineering 2 (1996) 259-286.

[7] C. Chen, Social networks at Sempra Energy's IT division are key to building strategic capabilities, Global Business and Organizational Excellence 26 (2007) 16-24.

[8] P.S. Chinowsky, J. Diekmann, J. O'Brien, Project organizations as social networks, Journal of Construction Engineering and Management 136 (2010) 452-458.

[9] N. Clarke, Transorganization development for network building, Journal of Applied Behavioral Science 41 (2005) 30-46.

[10]D.L. Cook, Program evaluation and review techniques, applications in education, U.S. Office of Education Cooperative Monograph 17 (OE-12024) (1966).

[11]R. Cross, S.P. Borgatti, A. Parker, Making invisible work visible: Using social network analysis to support strategic collaboration, California Management Review 44 (2002) 25-46.

[12]P. De Meo, A. Nocera, G. Terracina, D. Ursino, Recommendation of similar users, resources and social networks in a Social Internetworking Scenario, Information Sciences 181 (2011) 1285-1305.

[13]C. Durugbo, A. Tiwari, J.R. Alcock, Managing Information Flows for Product-Service Systems Delivery. Proceedings of the 2nd CIRP IPS2 Conference, Linköping, Sweden, 2010, pp. 365-370.

[14]M. Ehsani, A. Makui, S. Sadi Nezhad, A methodology for analyzing decision networks, based on information theory, European Journal of Operational Research 202 (2010) 853-863.

[15] S.D. Eppinger, Innovation at the speed of information, Harvard Business Review 79 (2001) 149-158, 178.

[16]D.G. Gregg, Designing for collective intelligence, Communications of the ACM 53 (2010) 134-138.

[17]F. Grippa, A social network scorecard to monitor knowledge flows across communication media, Knowledge Management Research and Practice 7 (2009) 317-328.

[18] J.-P. Hatala, J.G. Lutta, Managing information sharing within an organizational setting: A social network perspective, Performance Improvement Quarterly 21 (2009) 5-33.

[19] P. Hawe, C. Webster, A. Shiell, A glossary of terms for navigating the field of social network analysis, Journal of Epidemiology and Community Health 58 (2004) 971-975.

[20] D.W. Hsiao, A.J.C. Trappey, L. Ma, P.-S. Ho, An integrated platform of collaborative project management and silicon intellectual property management for IC design industry, Information Sciences 179 (2009) 2576-2590.

[21]M. Klein, C. Dellarocas, Knowledge-based approach to handling exceptions in workflow systems, Computer Supported Cooperative Work: CSCW: An International Journal 9 (2000) 399-412.

[22] M. Krauss, N. Mueller, D. Luke, Interorganizational relationships within state tobacco control networks: a social network analysis, Preventing chronic disease 1 (2004) A08.

[23] R. Kumar, Research methodology, Longman, London, 1996. 
[24] T. Kvan, Collaborative design: What is it? Automation in Construction, 9 (2000) 409-415.

[25] J.M. Lewis, J.I. Baeza, D. Alexander, Partnerships in primary care in Australia: Network structure, dynamics and sustainability, Social Science and Medicine 67 (2008) 280-291.

[26]L. López, J.F.F. Mendes, M.A.F. Sanjuán, Hierarchical social networks and information flow, Physica A: Statistical Mechanics and its Applications, 316 (2002) 695-708.

[27] M.L. Maher, A. Cicognani, S.J. Simoff, An experimental study of computer mediated collaborative design, International Journal of Design Computing 1 (1998) available online: http://wwwfaculty.arch.usyd.edu.au/kcdc/ijdc/vol01/papers/maherFrameset.htm

[28] J.A. Martin, K.M. Eisenhardt, Rewiring: Cross-business-unit collaborations in multibusiness organizations, Academy of Management Journal 53 (2010) 265-301.

[29] H.B. Milward, K.G. Provan, Measuring network structure, Public Administration 76 (1998) 387-407.

[30] M.S. Mizruchi, What do interlocks do? An analysis, critique and assessment of research on interlocking directorates, Annual Review of Sociology 22 (1996) 271-298.

[31]R. Monclar, A. Tecla, J. Oliveira, J.M. de Souza, MEK: Using spatial-temporal information to improve social networks and knowledge dissemination, Information Sciences 179 (2009) 2524-2537.

[32] M.E.J. Newman, The structure of scientific collaboration networks, Proceedings of the National Academy of Sciences of the United States of America 98 (2001) 404-409.

[33] Y. Ni, L. Xie, Z.-Q. Liu, Minimizing the expected complete influence time of a social network, Information Sciences 180 (2010) 2514-2527.

[34] A. Oberg, P. Walgenbach, Hierarchical structures of communication in a network organization, Scandinavian Journal of Management 24 (2008) 183-198.

[35]F. Pahng, N. Senin, D. Wallace, Distribution modelling and evaluation of product design problems, CAD Computer Aided Design 30 (1998) 411-423.

[36] S. Pryke, S. Pearson, Project governance: Case studies on financial incentives, Building Research and Information 34 (2006) 534-545.

[37]D. Richards, A social software/Web 2.0 approach to collaborative knowledge engineering, Information Sciences 179 (2009) 2515-2523

[38]B. Schultz-Jones, Examining information behavior through social networks: An interdisciplinary review, Journal of Documentation 65 (2009) 592-631

[39] N. Shyamsundar, R. Gadh, Collaborative virtual prototyping of product assemblies over the Internet, CAD Computer Aided Design 34 (2002) 755-768.

[40]D.H. Sonnenwald, Communication roles that support collaboration during the design process, Design Studies 17 (1996) 277-30.

[41] J. Stempfle, P. Badke-Schaub, Thinking in design teams - An analysis of team communication, Design Studies 23 (2002) 473-496.

[42] T.W. Valente, K.A. Coronges, Stevens, G.D., Cousineau, M.R., Collaboration and competition in a children's health initiative coalition: A network analysis, Evaluation and Program Planning 31 (2008) 392-402.

[43] W.M.P. Van Der Aalst, H.A. Reijers, M. Song, Discovering social networks from event logs, Computer Supported Cooperative Work: CSCW: An International Journal 14 (2005) 549-593.

[44]X.B. Wang, P.D.F. Kilduff, Structuring a concurrent design environment for effective product design and development, Journal of the Textile Institute Part 1: Fibre Science and Textile Technology 90 (1999) $83-91$.

[45]L. Wang, W. Shen, H. Xie, J. Neelamkavil, A. Pardasani, Collaborative Conceptual Design—state of art and future trends, CAD Computer-Aided Design 34 (2002) 981-996.

[46]L. White, Connecting organizations: developing the idea of network learning in inter-organizational settings, Systems Research and Behavioral Science 25 (2008) 701-716.

[47] H. Wi, S. Oh, J. Mun, M. Jung, A team formation model based on knowledge and collaboration, Expert Systems with Applications 36 (2009) 9121-9134.

[48]T. Wolf, A. Schröter, D. Damian, L.D. Panjer, T.H.D. Nguyen, Mining task-based social networks to explore collaboration in software teams, IEEE Software 26 (2009) 58-66. 
[49]F. Wu, B.A. Huberman, L.A. Adamic, J.R. Tyler, Information flow in social groups, Physica A: Statistical Mechanics and its Applications 337 (2004) 327-335.

[50]X.W. Xu, T. A. Liu, Web-enabled PDM system in a collaborative design environment, Robotics and Computer-Integrated Manufacturing 19 (2003) 315-328.

[51]A. Yassine, D. Falkenburg, K. Chelst, Engineering Design Management: An Information Structure Approach, International Journal of Production Research 37 (1999) 2957-2975.

[52] J. Zhu, J. Gong, W. Liu, T. Song, J. Zhang, A collaborative virtual geographic environment based on P2P and Grid technologies, Information Sciences 177 (2007) 4621-4633. 